\title{
Recycling of PVPP used in the wine industry: An opportunity for obtaining reusable PVPP and bioactive phenolic compounds
}

\author{
F. Cosme ${ }^{\text {a }, ~ S . S . ~ F e r r e i r a, ~ A . S . ~ A l v e s, ~ L . ~ F i l i p e-R i b e i r o, ~ a n d ~ F . M . ~ N u n e s ~}$ \\ Chemistry Research Centre - Vila Real (CQ-VR), Food and Wine Chemistry Lab, University of Trás-os-Montes and Alto Douro, \\ 5001-801 Vila Real, Portugal
}

\begin{abstract}
Polyvinylpolypyrrolidone (PVPP) is a synthetic water insoluble polymer produced by crosslinking of polyvinylpyrrilidone. It is used as a fining agent in the wine industry for removing low molecular weight phenolic compounds. There are no available data on the worldwide use of PVPP in the wine industry; nevertheless, its estimated use in Portugal in 2016 for white wine fining was nearly 147 tons. Used PVPP ends up on the municipal wastewater treatment plants, and as it contains significant quantities of adsorbed polyphenols it has a detrimental environmental impact. Recycling of PVPP for obtaining fully reusable PVPP and phenolic compounds for the food, cosmetic and pharmaceutical industry would increase the sustainability of its industrial use. Therefore, the purpose of this work was to develop a simple, cheap and eco-friendly regeneration strategy for the re-use of PVPP and at the same time to obtain pure extracts of wine phenolic compounds in order to exploit new opportunities for recycling used PVPP. The performance of the recycled PVPP for white wine fining was the identical when compared to a new PVPP.
\end{abstract}

\section{Introduction}

Awareness by consumers, producers and policy makers that the available resources are limited and of the negative environmental impact caused by the human activities, has been forcing industry to develop and adapt their manufacturing process towards more sustainable processes and products. The sustainability is achieved through development of economically viable and safer processes aiming minimizing the environmental impact of human activities. One of the issues causing serious concern is food waste, including wastes created during both processing and product distribution $[1,2]$. An important principle of the sustainability concept is the waste valorization [3], where wastes are a source of raw materials such as bioactive compounds, reducing their environmental impact [4]. One of the most interesting and important classes of these bioactive compounds are phenolic compounds. In fact the recovery of valuable compounds from agricultural and food industry wastes, namely phenolic compounds, is an emerging field with great potential to drive sustainable production [5-10]. It can be expected in the near future, upon of the European Commission's plans for a circular economy that manufacturers will need to demonstrate that their products have been designed to increase the possibility of reuse and recycling [11].

Polyvinylpolypyrrolidone (PVPP) is a water insoluble synthetic polymer obtained by cross-linking of polyvinylpyrrilidone (PVP) [12-14]. Due to its excellent adsorbent properties, selectivity, stability, inertness and non allergenicity [13] it is generally used in the production

\footnotetext{
a e-mail: fcosme@utad.pt
}

of wine, beer, juices and other beverages to avoid haze formation, eliminating compounds responsible for bitterness, astringency, browning and pinking [14,15]. Phenolic compounds bind to PVPP through hydrophobic interaction between the PVPP, specially its pyrrolidone ring and their aromatic ring and by hydrogen-bond between the phenol groups and carbonyl group of pyrrolidone [12,14-17]. It is more efficient at low $\mathrm{pH}$ due to the protonation of the adsorbate [15] although the structure of the polyphenol plays an important role for their interaction with PVPP [18]. It is widely used in the wine industry, being allowed to be applied in white and red wines with a maximum dose of $80 \mathrm{~g} / \mathrm{hL}$ [19]. Nowadays, most of the PVPP used in the wine industries ends on the municipal wastewater treatment plants. Although PVPP toxicity is low for the aquatic environment it biodegradability is slow [20]. Additionally, after application as it comprises significant quantities of adsorbed phenolic compounds its disposal can have a significant impact in the environmental, as phenolic compounds are very refractory [21].

The high commercial value of PVPP has raised the interest to recycling and reuse PVPP. In the beer industry a method has been developed for recycling and reusing PVPP obtained from haze removal from beer [22]. Therefore the main purpose of this work was to develop a simple, cheap and eco-friendly regeneration strategy for re-use of PVPP, using an ammoniacal solution of ethanol to desorb the phenolic compounds, after the fining process in white wine, to evaluate the recycling cycles number in the adsorption performance of PVPP used in wine fining and at the same time to obtain pure extracts of wine 
phenolic compounds in order to exploit new opportunities for recycling used PVPP.

\section{Material and methods}

\subsection{PVPP and wine sample}

An unused PVPP was supplied by SAI Enology Company $(100 \%$ grade, average particle size $110 \mu \mathrm{m})$ and the wine used to evaluate the influence of the PVPP recycling cycles number in the adsorption performance of PVPP used as fining agent, has the following characteristics: young white wine of 2017 vintage, from Lisbon (Portugal), alcohol content $12.5(\% \mathrm{v} / \mathrm{v})$, titratable acidity $5.7 \mathrm{~g} / \mathrm{L}$ (as tartaric acid), volatile acidity $0.33 \mathrm{~g} / \mathrm{L}$ (as acetic acid), $\mathrm{pH} 3.56$, free sulphur dioxide $42 \mathrm{mg} / \mathrm{L}$ and total sulphur dioxide $101 \mathrm{mg} / \mathrm{L}$.

\subsection{Fining experiments}

In each fining experiment, the wine was fined with $40 \mathrm{~g} / \mathrm{hL}$ of PVPP (new and recycled). Wine without addition of PVVP was used as a control. The wine was stored at room temperature ( 3 days) in sealed flasks and then centrifuged $(537.6 \mathrm{~g} ; 15 \mathrm{~min})$ for further analysis. Experiments were performed in duplicate.

\subsection{PVPP regeneration and recovering of adsorbed phenolic compounds}

$100 \mathrm{~g}$ of wet PVPPs were mixed with $100 \mathrm{~mL}$ of an ammoniacal solution of ethanol $(0.1 \mathrm{M})$, after $15 \mathrm{~min}$ at room temperature, the suspension was filtered, being the filtrates neutralized to $\mathrm{pH} \sim 6.5$ with acetic acid, the procedures was repeated. PVPP was washed with water and dried to be reused and the filtrates obtained in each PVPP recycled were analysed by RP-HPLC-DAD as described by Ferreira et al. [23].

\subsection{Determination and quantification of Individual phenolic compounds by RP-HPLC-DAD}

Phenolic compounds in wine and present in the filtrates obtained during each PVPP regeneration were analysed by RP-HPLC-DAD (Ultimate 3000, Dionex). A reverse phase $\mathrm{C} 18$ column (ACE $5,250 \mathrm{~mm} \times 4.6 \mathrm{~mm}, 5 \mu \mathrm{m}$; ACE, Scotland) was used. Mobile phase consisted of solvent A 5\% formic acid and solvent B methanol, the flow rate was $1.0 \mathrm{~mL} / \mathrm{min}$ and the temperature was maintained at $30^{\circ} \mathrm{C}$ during the run. The elution program was as follows: $5 \%$ B from zero to 5 min followed by a linear gradient up to $65 \%$ B until $65 \mathrm{~min}$ and from 65 to $67 \mathrm{~min}$ down to $5 \%$ B. Detection was performed by absorbance reading between $200-600 \mathrm{~nm}$ with a photo diode-array detector (PDA-100, Dionex) [24]. The injection volume was $25 \mu \mathrm{L}$ for the extracts and $50 \mu \mathrm{L}$ for the white wines. Peak identification was confirmed by retention time and UV-spectra comparison with those of authentic commercial standards. For the quantification a calibration curve was used in the range of $50-1000 \mathrm{mg} / \mathrm{L}$ for rutin and chlorogenic acid, in the range of $5-80 \mathrm{mg} / \mathrm{L}$ for (-)-epicatechin and resveratrol, $5-100 \mathrm{mg} / \mathrm{L}$ for gallic acid, prototecachuic acid, (+)-catechin, caffeic acid and ferulic acid. For those compounds which no standards

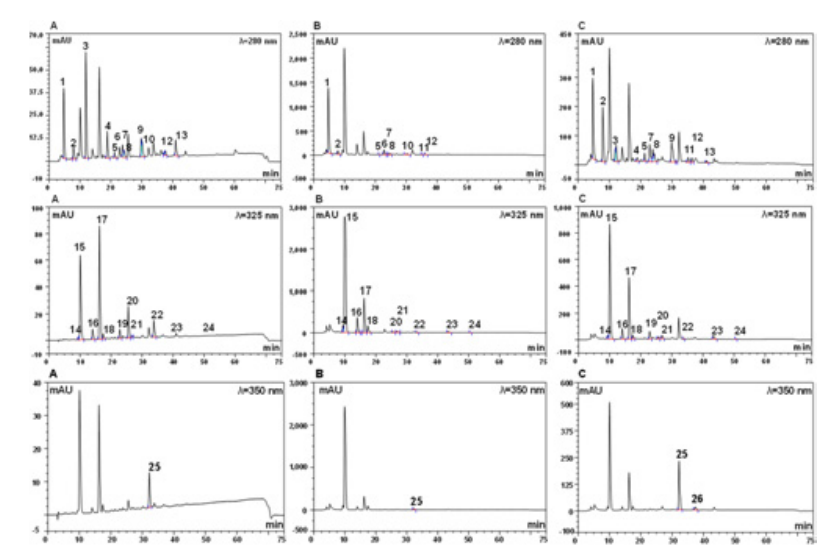

Figure 1. Chromatograms at $280 \mathrm{~nm}, 325 \mathrm{~nm}$ and $350 \mathrm{~nm}$ of phenolic compounds present in the first washing solution of trees different PVPP's (A, B, C). Peak identification: 1. gallic acid; 2. 3,4 dihydroxibenzoic; 3. (+) - catechin; 4. (-) - epicatechin; 5-8 and 11-13 unidentified derivative of procyanidins; 9. derivative of benzoic acid; 14. 2-S-glutathionyl caftaric acid; 15. trans-caftaric acid; 16. coutaric acid; 17. chlorogenic acid; 18. caffeic acid; 19. $p$-coumaric acid, 20. derivative glycosylated of resveratrol; 21. ferulic acid; 22. resveratrol; 23. caffeic acid ethyl ester; 24. ferulic acid ethyl ester; 25. rutin and 10, 26. unknown.

were available calibration curves of gallic acid, caffeic acid, $p$-coumaric acid and rutin were used for benzoic acid derivatives, cinnamic acids derivatives (caftaric and coutaric acids) and flavonol derivatives, respectively.

\subsection{Effect of the recycling cycles number in PVPP performance and recovery of adsorbed phenolic compounds}

The same wine was fined at least 4 times with the PVPP new/recycled and recovered phenolic compounds adsorbed was measured as described above, and the recovery rate was calculated.

\subsection{Statistical analysis}

One way ANOVA with a Tukey post-hoc test was used for indicating significant differences $(p<0.05)$ between more than two means. Analyses were performed using the Statistica 10 software. (Statsoft, OK, USA).

\section{Results and discussion}

In a previous study performed in our laboratory Ferreira et al. [23] observed that the use of an alkaline solution of ethanol allows to desorb the adsorbed phenolic compounds in yields ranging from $2.82 \mathrm{~g} / \mathrm{kg}$ of wet PVPP to $10.90 \mathrm{~g} / \mathrm{kg}$ of wet PVPP, using three different used PVPP's from the wine industry used in white wine fining for removing browning [25] and pinking phenolic compounds [26]. In this work were recovered 20 to 24 phenolic compounds, being the most abundant (+)-catechin, (-)-epicatechin, chlorogenic acid, trans-caftaric acid and gallic acid as shown in Fig. 1, the phenolic profile was determined by RP-HPLC-DAD.

Also the efficiency of the recycled PVPP (recycled for times) was compared with a new PVPP in the same white wine by sequential fining experiments. 
Table 1. Adsorption performance of white wine phenolic compounds of a new PVPP and the same PVPP after each recycling cycles.

\begin{tabular}{|l|c|}
\hline PVPP & g Total phenolic adsorbed/kg of PVPP \\
\hline New & $3.970 \pm 0.322$ \\
\hline $1^{\text {st }}$ recycling & $2.980 \pm 0.228$ \\
\hline $2^{\text {nd }}$ recycling & $2.479 \pm 0.223$ \\
\hline $3^{\text {rd }}$ recycling & $2.597 \pm 0.290$ \\
\hline $4^{\text {th }}$ recycling & $3.390 \pm 0.151$ \\
\hline
\end{tabular}

Fining performance

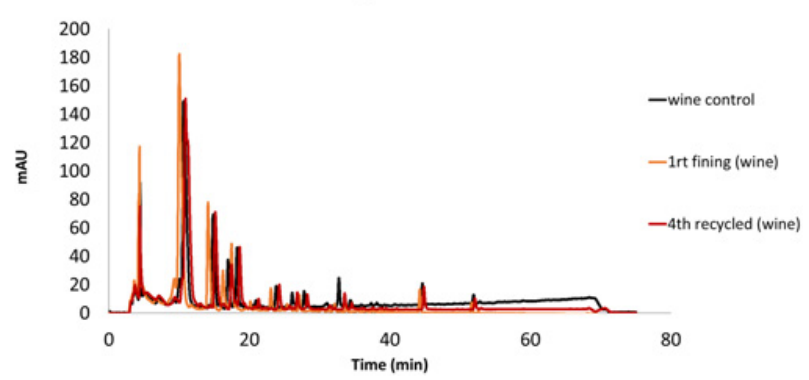

Figure 2. Fining performance of white wine phenolic compounds of a control wine using a new PVPP (first fining experiment) and the same PVPP after $4^{\text {th }}$ recycling cycles, obtained by RP-HPLCDAD.

\subsection{Effect of the recycling cycles number in PVPP fining performance and recovery of adsorbed phenolic compounds}

Table 1 shows the phenolic compounds adsorption performance after white wine fining with a new PVPP and the same PVPP after recycling four times, using the same white wine. The fining efficiency of the new PVPP was $3.97 \mathrm{~g}$ of total phenolic compounds by $\mathrm{kg}$ of PVPP, whereas the fining efficiency of the recycled PVPP until the $4^{\text {th }}$ cycle was not significantly different (ANOVA, $p<0.141$ ) from that of the new PVPP.

Regarding the phenolic compounds removal capacity of PVPP as shown in Fig. 2, (+)-catechin and resveratrol were removed to undetectable levels followed by rutin (74\% removal), protocatechuic acid (22\% removal), chlorogenic acid and caffeic acid ethyl ester (17\% removal), $p$-coumaric and ferulic acids (6\% removal), and trans-caftaric acid (5\% removal).

Being the recovery of the adsorbed phenolic compounds shown in Table 2, and for the new PVPP the percentage of recovered was on average $83.6 \pm 10.3 \%$ not being significantly different from $100 \%(p<0.266)$ and the recovery of the phenolic compounds adsorbed on the recycled PVPPs were not significantly different than those on the new PVPP (ANOVA, $p<0.139$ ). Rutin was the main recovered phenolic $(35 \% \mathrm{w} / \mathrm{w})$, followed by transcaftaric acid $(26 \% \mathrm{w} / \mathrm{w})$, chlorogenic acid $(16 \% \mathrm{w} / \mathrm{w})$, and coutaric acid $(6 \% \mathrm{w} / \mathrm{w})$, with the remaining phenolic compounds existing with an abundance less than $2 \%(\mathrm{w} / \mathrm{w})$ [23].

The results showed that the performance of the recycled PVPP for white wine fining was not significantly different from the performance of the new one, and the
Table 2. Recovery of white wine phenolic compounds adsorbed on a new PVPP and the same PVPP after each recycling cycles.

\begin{tabular}{|l|c|}
\hline PVPP & Recovery $\mathbf{( \% )}$ \\
\hline New & $83.630 \pm 10.300$ \\
\hline $1^{\text {st }}$ recycling & $75.500 \pm 7.600$ \\
\hline $2^{\text {nd }}$ recycling & $61.680 \pm 6.710$ \\
\hline $3^{\text {rd }}$ recycling & $65.530 \pm 7.850$ \\
\hline $4^{\text {th }}$ recycling & $81.800 \pm 7.500$ \\
\hline
\end{tabular}

PVPP can be recycled at least 4 times without loss of fining performance.

\section{Conclusions}

The results obtained in this work show that it is possible use recycled PVPP for wine fining with identical performance to the new one by applying a low cost and eco-friendly procedure that allows to reuse PVPP at least 4 times without changing the adsorption and the efficiency of the PVPP. At the same time, reused PVPP could decrease its synthesis and decrease the disposal in wastewater, decreasing the phenolic load of the effluents generated by the wine industry and obtaining in the same process valuable and pure phenolic compounds with high antioxidant activity and bioactivity, appreciated by other industries such as food, cosmetic and pharmaceutical industries.

This research was supported by European Investment Funds by FEDER/COMPETE/POCI under POCI-01-0145-FEDER007728 and funds from the Portuguese Foundation for Science and Technology (FCT) to CQ-VR (PEst-OE/QUI/UI0616/2014). This study has received funding from FEDER, Interreg EspanaPortugal Programme, under the framework of the Project ref 0377_IBERPHENOL_6_E. S.S.F. acknowledges the financial support provided by the European Social Funds and the Regional Operational Programme Norte 2020 (operation NORTE-085369-FSE-000054).

\section{References}

[1] A. Segre, Total Food: Sustainability of the AgriFood Chain, edited by K.W. Waldron, G.K. Moates, C.B. Faulds (The Royal Society of Chemistry, 2009), p. 162

[2] A. Segre, S. Gaiani (Royal Society of Chemistry Publishing, UK, 2011)

[3] P. Glavič, R. Lukman, J. Clean. Prod. 15, 1875 (2007)

[4] R.A.D. Arancon, C.S.K. Lin, K.M. Chan, T.H. Kwan, R. Luque, Energy Sci. Eng. 1, 53 (2013)

[5] S. Yusoff, J. Cleaner. Prod. 14, 87 (2006)

[6] D. Sud, G. Mahajan, M. Kaur, Bioresour. Technol. 99, 6017 (2008)

[7] A. Bhatnagar, M. Sillanpää, Chem. Eng. J. 157, 277 (2010).

[8] M. Koller, R. Bona, G. Braunegg, C. Hermann, P. Horvat, M. Kroutil, J. Martinz, J. Neto, L. Pereira, P. Varila, Biomacromolecules 6, 561 (2005)

[9] P.S. Kulkarni, C. Brazinha, C.A. Afonso, J.G. Crespo, Green Chem. 12, 1990 (2010)

[10] M.W. Nam, J. Zhao, M.S. Lee, J.H. Jeong, J. Lee, Green Chem. 17, 1718 (2015) 
[11] J.H. Clark, T.J. Farmer, L. Herrero-Davila, J. Sherwood, Green Chem. 18, 3914 (2016)

[12] L.W. Doner, G. Bécard, P.L. Irwin, J. Agric. Food Chem. 41, 753 (1993)

[13] C. Folch-Cano, C. Olea-Azar, H. Speisky, Colloids Surf. A 418, 105 (2013)

[14] P.J. Magalhães, J.S. Vieira, L.M. Gonçalves, J.G. Pacheco, L.F. Guido, A.A., Barros, J. Chromatogr. A 217, 3258 (2010)

[15] L. Jankowiak, I.V. Avermaete, R. Boom, A.J.V.D. Goot, Sep. Purif. Technol. 149, 479 (2015)

[16] Z-B. Dong, Y-R. Liang, F-Y. Fan, J-H. Ye, X-Q. Zheng, J-L. Lu, J. Agric. Food Chem. 59, 4238 (2011)

[17] B. Laborde, V. Moine-Ledoux, T. Richard, C. Saucier, D. Dubourdieu, J-P. Monti, J. Agric. Food Chem. 54, 4383 (2006)

[18] C. Morge, Revue des OEnologues 140, 22 (2011)

[19] Regulation (EU) No 1169/2011, of the European Parliament and of the Council, of 25 October 2011, Official Journal of the European Union L 304 (2011)
[20] Regulation (EC) 1907/2006, of the European Parliament and of the Council, of 18 December 2006, Official Journal of the European Union L 136 (2007)

[21] S. Kuppusamy, P. Thavamani, M. Megharaj, R. Naidu, Env. Tech. Inn. 4, 17 (2015)

[22] T.R. Noordman, D.N.M. Van, A. Richter, EP2595723 A1, 2010; Heineken Supply Chain B.V., EP20110738065, 2013

[23] S.S. Ferreira, A.J. Alves, L. Filipe-Ribeiro, F. Cosme, F.M. Nunes, ACS Sustainable Chem. Eng. 6, 4599 (2018)

[24] R. Guise, L. Filipe-Ribeiro, D. Nascimento, O. Bessa, F.M. Nunes, F. Cosme, Food Chem. 156, 250 (2014)

[25] F. Cosme, I. Capao, L. Filipe-Ribeiro, R.N. Bennett, A. Mendes-Faia, LWT-Food Sci. Technol. 46, 382 (2012)

[26] J. Andrea-Silva, F. Cosme, L. Filipe-Ribeiro, A.S.P. Moreira, A.C. Malheiro, M.A. Coimbra, M.R.M. Domingues, F.M. Nunes, J. Agric. Food Chem. 62, 5651 (2014) 